\title{
Contrôle du convertisseur monophasé par réseau de
} neurones

\section{Brahim Minaoui ${ }^{*}$}

Faculté des sciences et techniques, Université Cadi Ayyad, B.P. 523 Beni-Mellal, Maroc

(Reçu le 04 Mai 2006, accepté le 07 Décembre 2006)

*Correspondance, courriel : min@yahoo.fr

\section{Résumé}

La modélisation interne du convertisseur monophasé a montré qu'il est un système non linéaire dont la représentation interne est multi modèles. Ainsi pour commander la puissance électrique à sa sortie, nous avons utilisé un contrôleur non linéaire construit par un réseau de neurones multicouches (RNM).

Mots-clés : Contrôleur non linéaire, système multi modèles, réseau de neurones multicouches, apprentissage par rétro propagation.

\section{Abstract \\ Control of the single-phase converter using neural networks}

The single-phase converter (AC/DC) is a nonlinear multimodal system. In order to control the electrical power at the output of this system a direct nonlinear controller based on multilayer neural networks (MNN) is used.

Keywords : Nonlinear controller, multimodal system, multilayer neural network, learning and back propagation. 


\section{Introduction}

Les réseaux de neurones sont des approches inspirées du système nerveux. Leur utilisation pour la modélisation des fonctions mathématiques a fait l'objet de plusieurs travaux de recherche [1-3]. Ces études ont montré que les modèles connexionnistes sont capables de reproduire toute fonction par apprentissage.

Cette capacité d'apprendre des fonctions variées, qu'il n'est pas nécessaire de connaître analytiquement, a permis aux réseaux de neurones de trouver des champs d'application très variés en particulier la modélisation des contrôleurs non linéaires [4-7].

Dans le présent travail nous avons utilisé un réseau de neurones multicouches (RNM) pour construire un contrôleur non linéaire de la puissance électrique à la sortie du convertisseur monophasé qui est un système non linéaire.

\section{Principe}

\section{2-1. Description du convertisseur monophasé}

Le convertisseur monophasé étudié est représenté par la Figure 1. II est formé par un générateur de tension sinusoïdale $E(t)=E_{0}$ sinct, d'un circuit de commande à triac, d'un transformateur monophasé, d'un pont redresseur et d'un filtre passe-bas. La sortie $\mathrm{s}(\mathrm{t})$ de ce convertisseur est indiquée par la Figure 2.

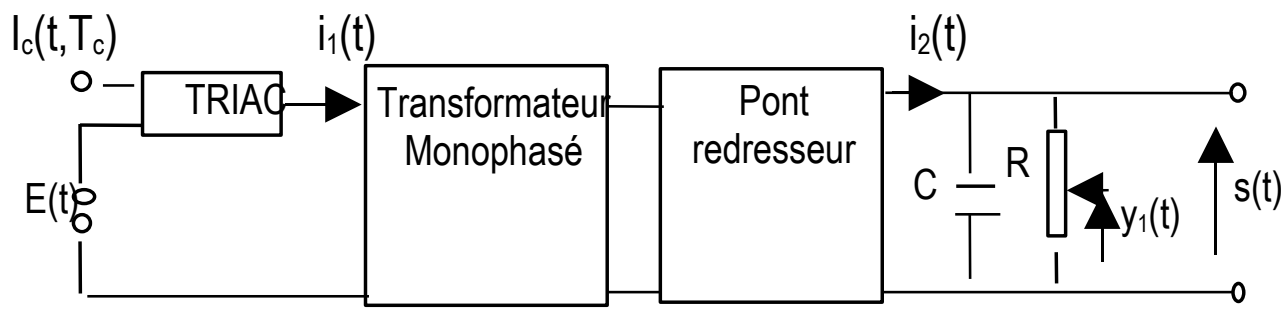

Figure 1: schéma synoptique du convertisseur monophasé 
Une impulsion du signal $I_{c}\left(t, T_{c}\right)$ d'amplitude et de durée $\mathrm{T}$ prédéfinies, appliquée au circuit de commande à l'instant $t=T_{c}$ modulo $T / 2\left(T_{c}\right.$ est définie par rapport à tout passage de $E(t)$ par zéro), provoque le fonctionnement du convertisseur monophasé pendant une partie de chaque alternance de $E(t)$. Le convertisseur et sa charge consomment l'énergie fournie par l'entrée. $A t=T_{1}$ modulo $T / 2$, instant dépendant des constantes du circuit, l'entrée se bloque. II n'y a pas de consommation d'énergie à l'entrée. La charge consomme l'énergie résiduelle dans l'étage du filtrage jusqu'à l'arrivée d'une nouvelle impulsion.

La durée $T_{1}$ est toujours comprise entre $T_{0}$ et $T_{1}$. $T_{0}$ correspond à la commutation, en absence du circuit de commande, du modèle exponentiel au modèle sinusoïdal. La puissance à la sortie de ce système atteint sa valeur maximale pour $T_{c}=T_{0}$ et sa valeur minimale pour $T_{c}=T_{1}$. Le contrôle de cette puissance n'est donc possible que pour des puissances moyennes comprises entre ces deux valeurs limites.

\section{2-2. Modélisation interne}

L'étude de ce montage a montré que la sortie $s(t) d u$ convertisseur monophasé passe pour chaque alternance d'une évolution sinusoïdale $s(t)=s_{s}(t)$ à une autre exponentielle $s(t)=s_{\text {ex }}(t)$ provenant de l'isolement $d u$ filtre passe-bas ou inversement. L'état d'évolution de ce système est représenté par deux modèles à savoir [8]:

- Le modèle sinusoïdal $\mathrm{s}(\mathrm{t})=\mathrm{s}_{\mathrm{s}}(\mathrm{t})$ pour $\mathrm{t} \in\left[T_{c}, T_{1} \mid\right.$

$$
\begin{aligned}
& \frac{d X(t)}{d t}=A(R) X(t)+B E_{o}|\sin \omega t|+K \\
& y(t)=H_{s} X(t)
\end{aligned}
$$

- Le modèle exponentiel $\mathrm{s}(\mathrm{t})=\mathrm{s}_{\mathrm{ex}}(\mathrm{t})$ pour $\mathrm{t} t \in\left[0, T_{c} \mid\right.$ ou $\mathrm{t} \in\left[T_{1}, \frac{T}{2}[\right.$

$$
\begin{aligned}
& \frac{d X(t)}{d t}=A(R) X(t)+B E_{o}|\sin \omega t|+K \\
& y(t)=H_{e} X(t)
\end{aligned}
$$


Avec $X(t)=\left|x_{1}(t), x_{2}(t), x_{3}(t), x_{4}(t)\right|^{T}=\left|s_{s}(t), \dot{i}_{1}(t), \dot{i}_{2}(t), s_{e x}(t)\right|^{T}$ est le vecteur d'état interne; $y(t)=y_{1}(t)$ est l' observation; $A(R), B, K, H_{e}$ et $H_{s}$ sont définis par les matrices suivantes [8]:

$$
\begin{aligned}
& \mathrm{A}=\left[\begin{array}{cccc}
-\frac{1}{R C} & 0 & \frac{1}{C} & 0 \\
\frac{M}{L_{1}^{\prime} L_{2}^{\prime}-M^{2}} & \frac{-L_{2}^{\prime}\left(r_{T}+r_{1}\right)}{L_{1}^{\prime} L_{2}^{\prime} M^{2}} & \frac{M\left(2 r_{D}+r_{2}\right)}{L_{1}^{\prime} L_{2}^{\prime}-M^{2}} & 0 \\
\frac{-L_{1}^{\prime}}{L_{1}^{\prime} L_{2}^{\prime}-M^{2}} & \frac{M\left(r_{T}+r_{1}\right)}{L_{1}^{\prime} L_{2}^{\prime}-M^{2}} & \frac{-L_{1}^{\prime}\left(2 r_{D}+r_{2}\right)}{L_{1}^{\prime} L_{2}^{\prime}-M^{2}} & 0 \\
0 & 0 & 0 & \frac{-1}{R C}
\end{array}\right] \\
& \mathrm{B}=\left[\begin{array}{c}
0 \\
\frac{L_{2}^{\prime}}{L_{1}^{\prime} L_{2}^{\prime}-M^{2}} \\
\frac{-M}{L_{1}^{\prime} L_{2}^{\prime}-M^{2}} \\
0
\end{array}\right], \mathrm{K}=\left[\begin{array}{c}
0 \\
\frac{2 M v_{D}-L_{2}^{\prime} v_{T}}{L_{1}^{\prime} L_{2}^{\prime}-M^{2}} \\
\frac{M v_{T}-2 L_{1}^{\prime} v_{D}}{L_{1}^{\prime} L_{2}^{\prime}-M^{2}} \\
0
\end{array}\right] \\
& \mathrm{H}_{\mathrm{s}}=\left[\begin{array}{llll}
\alpha & 0 & 0 & 0
\end{array}\right]^{T}, \mathrm{H}_{\mathrm{e}}=\left[\begin{array}{llll}
0 & 0 & 0 & \alpha
\end{array}\right]^{T}
\end{aligned}
$$

Où $\left(\mathrm{V}_{T}, \mathrm{r}_{\mathrm{T}}\right)$ sont les paramètres du triac, $\left(\mathrm{V}_{\mathrm{d}}, \mathrm{r}_{\mathrm{d}}\right)$ les paramètres des diodes du redresseur et $\left(r_{1}, r_{2}, L_{1}^{\prime}, L_{2}^{\prime}, M\right)$ ceux du transformateur monophasé tenant compte des fuites et pertes. $C$ et $R$ deux grandeurs représentant respectivement la capacité du filtre utilisé et la résistance équivalente à l'impédance de charge du convertisseur.

$E_{0}$ et $R$ sont des paramètres variables figurant dans les coefficients des équations relatives au deux modèles indiqués. Le processus physique est donc décrit par un système non linéaire entre les variables d'état et les variables de fluctuations aléatoires. Ainsi le système physique présente deux propriétés qui le différencient nettement des systèmes relevant de l'automatisme linéaire. En effet, ce système physique change de modélisation au cours de temps, en plus chaque modèle est non linéaire. La commande de ce système nécessite alors un contrôleur non linéaire. Pour ce faire, nous avons recouru aux réseaux de neurones. 


\section{2-3. Présentation de la commande non linéaire adoptée}

Nous avons utilisé un réseau de neurones multicouches (RNM) pour construire un contrôleur non linéaire. La structure générale adoptée pour réaliser la commande du système considéré, est représentée par la Figure2.

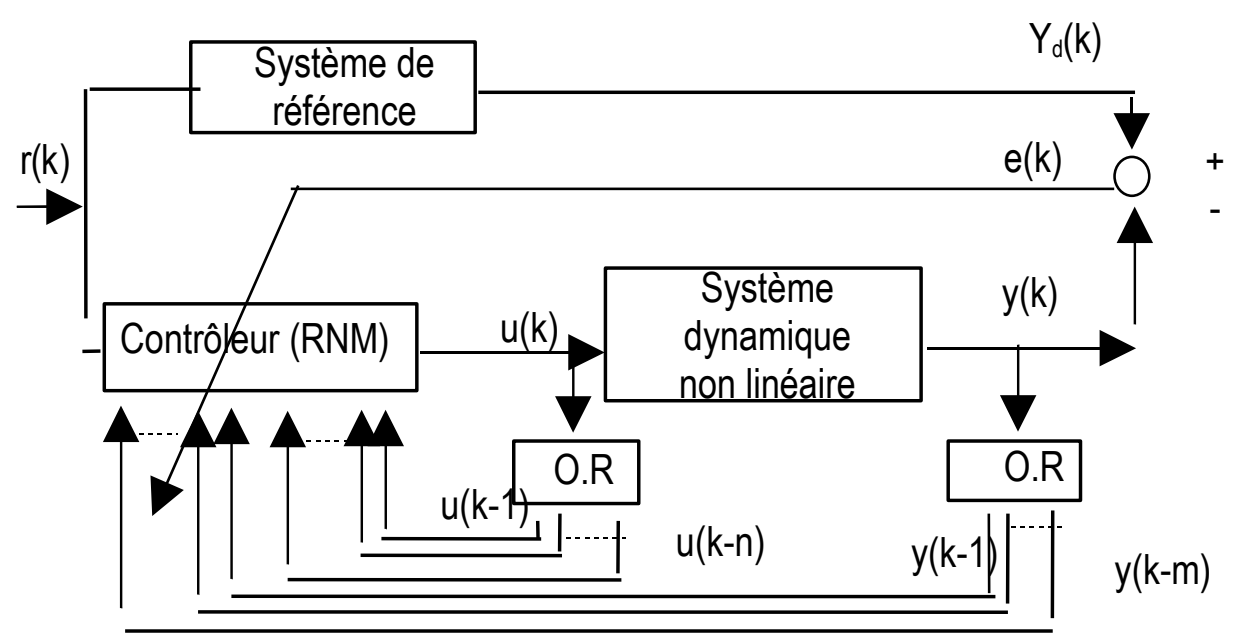

Figure 2: Commande adaptative directe d'un système non linéaire (O.R : opérateur de retard)

\section{2-4. Structure du contrôleur (RNM)}

Le contrôleur utilisé est constitué par un réseau de neurones multicouches (RNM) dont la structure est représentée à la Figure 3.

Les neurones (unités) de ce réseau sont organisés en quatre couches (niveaux) à savoir:

- une couche d'entrée contenant $n_{\mathrm{e}}$ neurones

- deux couches cachées $\mathrm{Cc}_{1}$ et $\mathrm{cc}_{2}$ comportant respectivement $\mathrm{nc}_{1}$ et $\mathrm{nc}_{2}$ neurones

- une couche de sortie constituée par un seul neurone.

Chaque neurone de chaque couche est connecté à tous les neurones de la couche suivante. 
L'entrée est fixée à 1 et correspond à l'unité de seuil ou de biais 'bias unit'.

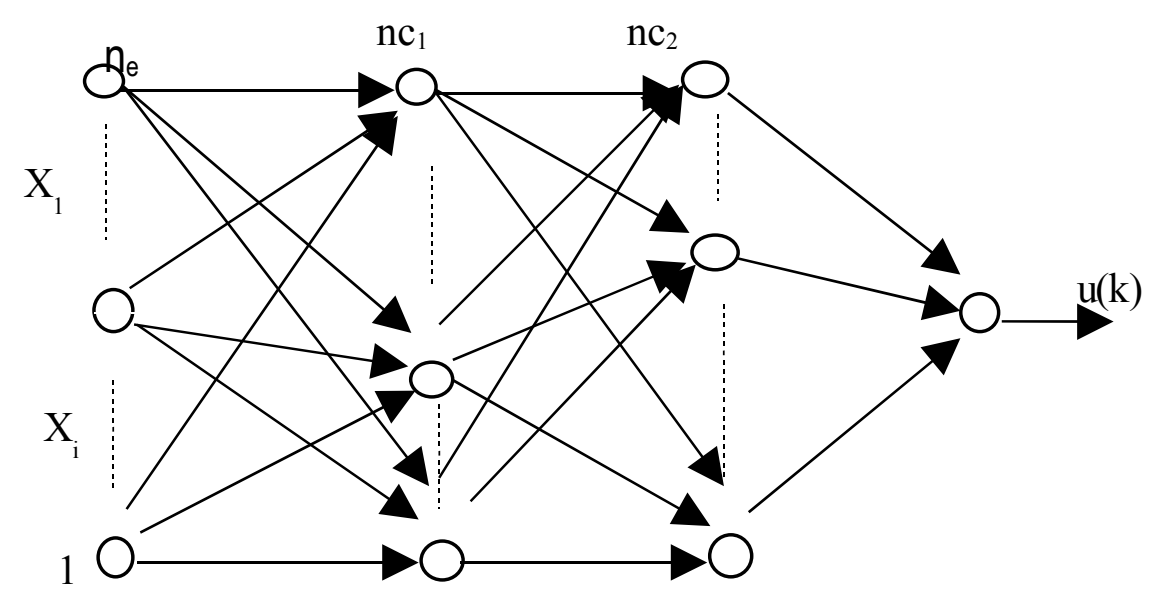

Figure 3 : Réseau de neurones à quatre couches

Soit et les poids des différentes connexions entre les neurones du réseau considéré. La relation entre son entrée $\mathrm{x}=\left[x_{1}, x_{2}, \ldots, x_{n e}\right]^{T}$ et sa sortie $u(k)$ est définie par les équations (3), (4) et (5) [9] :

$$
\begin{array}{ll}
u(k)=\sum_{l=1}^{n c_{2}} W_{l}^{z} Z_{l}(k) & \mathrm{Z}_{1}(k)=H\left(Y_{l}^{S}(k)\right) \\
Y_{l}^{S}(k)=\sum_{j=1}^{n c_{1}} W_{j l}^{y} Y_{j}(k) & \mathrm{Y}_{\mathrm{j}}(k)=H\left(X_{j}^{S}(k)\right) \\
X_{j}^{S}(k)=\sum_{i=1}^{n e} W_{i j}^{x} x_{i}(k) &
\end{array}
$$

Avec $H(x)=\frac{1}{1+\exp (-x)}$ la fonction sigmoïde, une fonction différentiable et croissante, représentant la loi d'activation des neurones.

\section{2-5. Algorithme d'apprentissage}

Nous avons recouru à l'apprentissage par retropropagation qui consiste à minimiser l'erreur suivante [10]: 


$$
\mathrm{J}(\mathrm{k})=\frac{1}{2} \lambda e^{2}+(1-\lambda) \frac{1}{\beta} \ln (\cosh (\beta e))(1-\lambda) \frac{1}{\beta} \ln (\cosh (\beta e))
$$

ou $e=u_{d}(k)-u(k)$ avec $u_{d}(k)$ la sortie désirée. $\left.\left.\lambda \in\right] 0,1\right]$ et $\beta>0$

On va minimiser cette erreur par la méthode du gradient, c'est-à-dire en faisant évoluer les poids dans la direction indiquée par le gradient de $\mathrm{J}$ :

$$
\Delta W(k+1)=-\alpha \frac{\partial J}{\partial W}
$$

où est le taux d'apprentissage

$\operatorname{Or} \frac{\partial J}{\partial W}=\frac{\partial J}{\partial e} \frac{\partial e}{\partial W}=-g \frac{\partial u(k)}{\partial W}$

Où $\quad g=\lambda e+(1-\lambda) \tanh (\beta e)$

La dérivée de $\mathrm{u}(\mathrm{k})$ par $W_{l}^{Z}, W_{j l}^{Y}$ et $W_{i j}^{X}$ permet d' obtenir respectivement les dérivées partielles suivantes:

$$
\begin{aligned}
& \frac{\partial u(k)}{\partial W_{l}^{Z}}=Z_{l}(k) \\
& \frac{\partial u(k)}{\partial W_{j l}^{y}}=W_{l}^{Z} \frac{\partial Z_{l}(k)}{\partial W_{j l}^{y}}==W_{l}^{Z} H^{\prime}\left(Y_{l}^{S}(k)\right) Y_{j}(k) \\
& \frac{\partial u(k)}{\partial W_{i j}^{x}}=\sum_{l=1}^{n c_{2}} W_{l}^{Z} \frac{\partial Z_{l}(k)}{\partial W_{i j}^{x}}=\sum_{l=1}^{n c_{2}} W_{l}^{Z} H^{\prime}\left(Y_{l}^{s}(k)\right) W_{j l}^{y} \frac{\partial Y_{j}(k)}{\partial W_{i j}^{x}} \\
& =\sum_{l=1}^{n c_{2}} W_{l}^{Z} H^{\prime}\left(Y_{l}^{s}(k)\right) W_{j l}^{y} \cdot H^{\prime}\left(X_{j}^{s}(k)\right) x_{i}(k) \\
& H^{\prime}\left(Y_{l}^{s}(k)\right)=Z_{l}(k)\left(1-Z_{l}(k)\right) \quad \text { et } \mathrm{H}^{\prime}\left(\mathrm{X}_{\mathrm{j}}^{\mathrm{S}}(k)=Y_{j}(k)\left(1-Y_{j}(k)\right)\right.
\end{aligned}
$$

Par conséquent les équations (7), (8), (9), (10), (11) et (12) permettent d'obtenir les équations de l'estimateur récursif suivantes: 


$$
\begin{aligned}
& W_{l}^{Z}(k+1)=W_{l}^{z}(k)+\alpha \cdot g \cdot Z_{l}(k) \\
& W_{j l}^{y}(k+1)=W_{j l}^{y}(k)+\alpha \cdot g \cdot W_{l}^{z} \cdot H^{\prime}\left(Y_{l}^{s}(k) \cdot Y_{j}(k)\right. \\
& W_{i j}^{x}(k+1)=W_{i j}^{x}(k)+\alpha \cdot g \cdot \sum_{l=1}^{n c_{2}} W_{l}^{Z} H^{\prime}\left(Y_{l}^{S}(k)\right) W_{j l}^{y} \cdot H^{\prime}\left(X_{j}^{S}(\mathrm{k})\right) \mathrm{x}_{\mathrm{i}}(\mathrm{k})
\end{aligned}
$$

L'objet du contrôleur, constitué par RNM, est d'apprendre la loi de commande:

$$
u(k)=R N M[u(k-1), \ldots u(k-n), r(k), y(k-1), \ldots, y(k-(m))] .
$$

Mais pour cet apprentissage, le signal d'erreur n'est pas calculé sur la commande mais sur sa séquence, qui est l'écart de $\mathrm{y}(\mathrm{k})$ à la consigne $y_{d}(k) .\left(e=y_{d}(k)-y(k)\right)$.

\section{Simulation}

La puissance électrique moyenne à commander est évaluée sur chaque alternance par $\mathrm{N}$ valeurs de la tension de sortie $\mathrm{s}(\mathrm{t})$ du convertisseur. Pour la énième alternance on a [11]:

$\mathrm{P}(\mathrm{n})=\frac{1}{2 N R} \sum_{k=1}^{N}\left[s^{2}\left((n-1) \frac{T}{2}+k T e\right)+s^{2}\left((n-1) \frac{T}{2}+(k+1) T e\right)\right]$

Cette puissance dépend de plusieurs paramètres en particulier de $T_{c}$ (durée de blocage du triac pendant chaque alternance de l'entrée), de $E_{0}$ (tension efficace d'entrée) et de $\mathrm{R}$ (résistance équivalente de la charge du convertisseur). La connaissance à tout instant de ces paramètres permet de commander la puissance moyenne à la sortie.

En effet, dans un premier temps nous avons étudié le contrôle du convertisseur en fixant $E_{0}$ et $R$ à des valeurs constantes. A partir de l'entrée $X(n)=\left[T_{c}(n-1), T_{c}(n-2), r(n), P(n-1), P(n-2), 1\right]$, la loi de commande $T_{c}(n)$ est restituée par le réseau de neurones RNM. Ensuite la puissance $P(n)$ à la sortie du système est calculée par l'équation (14) et comparée à la puissance désirée $P_{\mathrm{d}}$. La différence est soumise au RNM pour corriger les poids des différentes connexions afin d'obtenir la meilleure loi de commande $T_{c}$. 
Après plusieurs essais, le nombre de neurones de la première et de la deuxième couche cachée est fixé respectivement à $\mathrm{nc}_{1}=20$ et $\mathrm{nc}_{2}=10$.

Ensuite, nous avons refait l'étude précédente en tenant compte des variations de $R$ et de $E_{0}$. La perturbation de ces paramètres est simulée par deux processus auto-régréssif aléatoires de variance respectives et. Ils permettent de générer les valeurs probables de $E_{0}$ et de $R$ initialisées à priori.

\section{Résultats}

Les résultats de la simulation sont illustrés par les exemples donnés aux Figures 4 et 5 . Ces exemples sont choisis pour mettre en évidence les performances de la commande numérique non linéaire utilisée en présentant le cas où $E_{0}$ et $R$ sont fixées respectivement à $235 \mathrm{~V}$ et $1 \mathrm{~K}$; et le cas où $E_{0}$ et $R$ sont assez bruitées avec 8.4 et 0.99

La Figure 4 révèle que la régulation de la puissance électrique moyenne à la sortie du convertisseur monophasé, est assurée. Le temps de réponse est $t_{r}=0.01 \mathrm{~s}$ et le taux d'erreur est $T_{\text {err }}=0$ après la durée $t_{r}$.

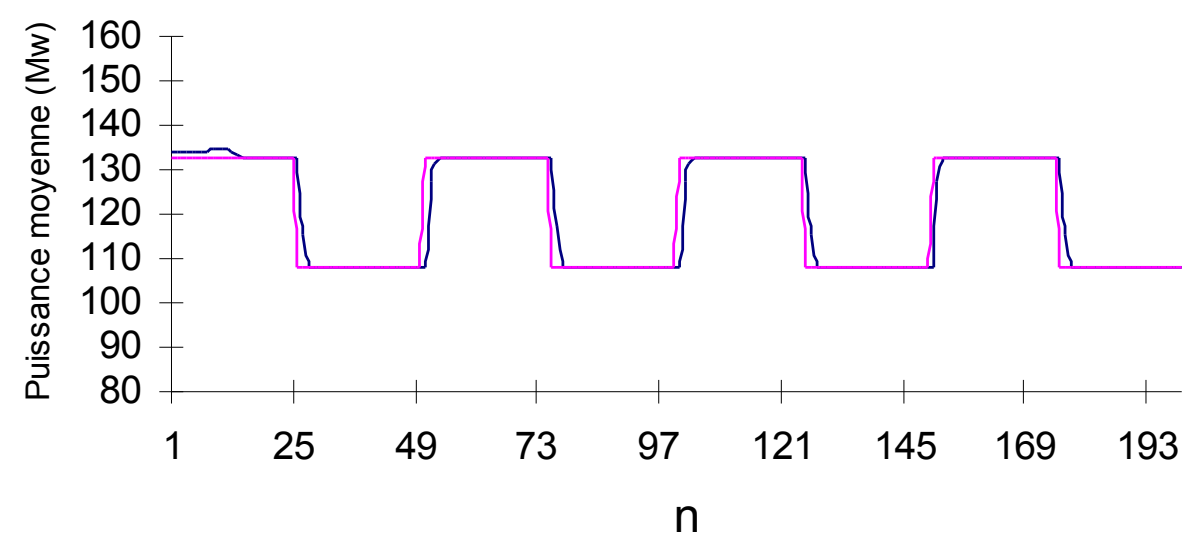

Figure 4 : Poursuite de la puissance moyenne $P(-)$ à sa consigne $P_{d}(-)$. Avec $E_{0}=235 \mathrm{~V}$ et $R=1 \mathrm{~K}$

D'après la Figure 5, on constate que malgré les variations aléatoires de la tension $E_{0}$ et de la charge $R$ la puissance moyenne $P$ poursuit sa 
valeur de consigne $P_{d}$. Le temps de réponse est $t_{r}=0.02 \mathrm{~s}$ et le taux d'erreur $T_{\text {er }} \leq 3 \%$ après la durée $t_{\text {r. }}$.

La comparaison des valeurs du temps de réponse $t_{r} \leq 0.02 s$ et du taux d'erreur $\mathrm{T}_{\text {err }} \leq 3 \%$ obtenus dans ce travail par rapport à ceux obtenus dans les travaux antérieures [8] (commande analogique par $\mathrm{PI} \mathrm{t}_{\mathrm{r}}=0.70 \mathrm{~s}$ et $\mathrm{T}_{\text {err }} \leq 12 \%$, commande analogique par PID $\mathrm{t}_{\mathrm{r}}=3 \mathrm{~s}$ et $\mathrm{T}_{\text {err }} \leq 10 \%$ et commande numérique $\mathrm{t}_{\mathrm{r}}=0.01 \mathrm{~s}$ et $\mathrm{T}_{\text {err }} \leq 5 \%$ ) montre que la commande du système considéré par réseau de neurones RNM est satisfaisante.

Ces résultats obtenus par simulation, nous ont conduit à la réalisation pratique de la commande de la puissance électrique à la sortie du convertisseur monophasé en implémentant le contrôleur (RNM) sur un processeur spécialisé en traitement numérique du signal (Digital signal processor : DSP) $[12,13]$.

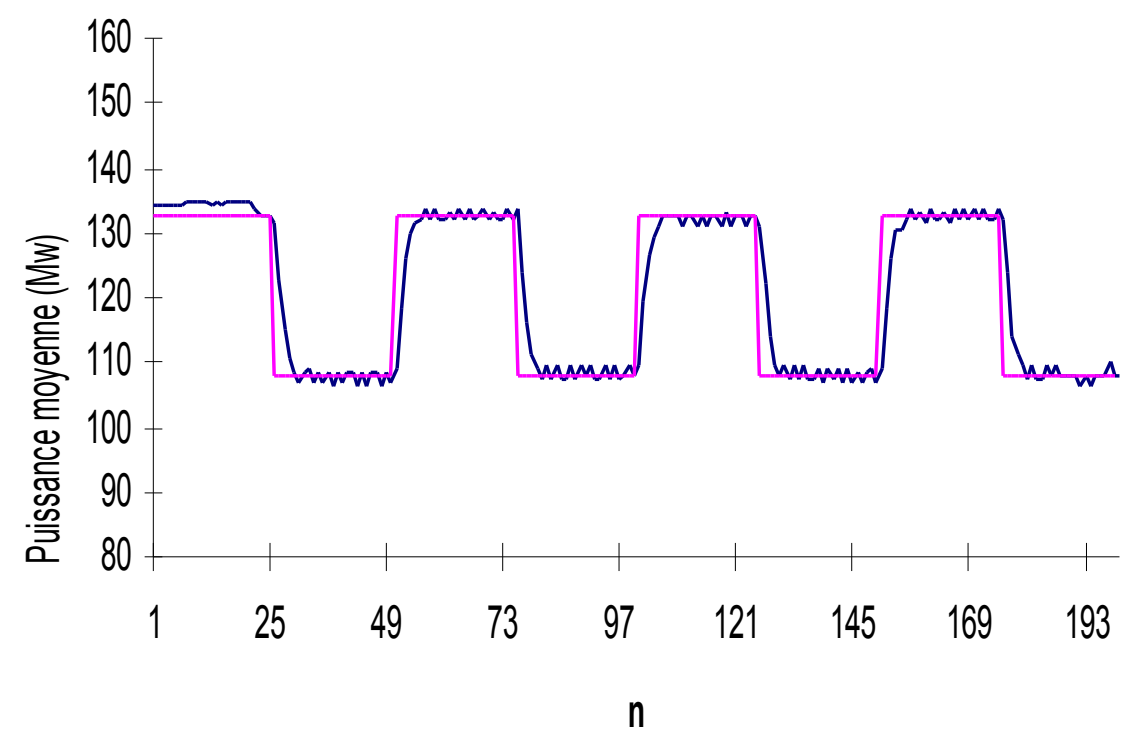

Figure 5 : Poursuite de la puissance moyenne $P(-)$ à sa consigne $P_{d}(-)$ en tenant compte des variations aléatoires de $E_{0}$ et $R$ $\operatorname{avec} E_{0}(t=0)=235 \mathrm{~V}, R(t=0)=1 \mathrm{~K},=8.4 \mathrm{et}=0.99$ 


\section{Réalisation pratique}

\section{5-1. Matériel utilisé}

Le matériel utilisé contient essentiellement :

* Un circuit analogique relatif au convertisseur monophasé donné à la Figure 1;

* Un amplificateur de courant, réalisé par un étage à transistors, permettant d'amplifier l'impulsion $I_{c}\left(t, T_{c}\right)$ délivré par le DSP. Ce courant amplifié est appliqué à la gâchette du triac ;

* Une carte qui reçoit la sortie du convertisseur monophasé et délivre l'impulsion $\mathrm{I}_{\mathrm{c}}\left(\mathrm{t}, \mathrm{T}_{\mathrm{c}}\right)$. Cette carte est réalisée autour $\mathrm{du}$ processeur TMS320C31 qui est spécialisé en traitement numérique du signal (DSP).

\section{5-2. Implémentation du contrôleur sur le DSP [11]}

L'implémentation du contrôleur (RNM) sur le DSP consiste à programmer les équations (3), (4) et (5) d'entrée/sortie du contrôleur neuronal, les équations (13) de l'estimateur récursif des poids des connexions neuronales et l'expression d'estimation de la puissance électrique moyenne (14) en langage assembleur du TMS320C31.

\section{5-3. Mise en œuvre du système de commande}

Pour mettre en œuvre le système de commande en temps réel nous mettons le convertisseur monophasé, la carte DSP et l'amplificateur de courant sous tension, ensuite nous chargeons le programme décrit précédemment dans la RAM du microprocesseur, puis nous exécutons ce programme.

La puissance électrique moyenne calculée par l'expression (14) est visualisée sur l'oscilloscope. Les résultats de la poursuite de cette puissance à sa consigne $P_{d}$ programmée sont satisfaisants. 


\section{Conclusion}

Dans ce travail nous avons montré la faisabilité d'une méthode de commande non linéaire par réseau de neurones en l'appliquant au convertisseur monophasé. Cette application trouve une intense utilisation dans les systèmes fonctionnant sous tension continue, à savoir en particulier les machines de chauffage par faisceau électronique ( systèmes de coévaporation) et celles fabriquées à base du moteur à courant continu.

\section{Références}

[1] - L. F. Mingo Lopez, " non-linear combination of neuro-computed basis to approximate functions ", International Journal of computer research, vol. 10, $n^{\circ} 2$ ( 2001) 223-226

[2] - P. Cardaliaguet and G. Euvrard, "Approximation of a function and its derivative with a neural network ", Neural networks, vol.5, (1992) 207-220

[3] - J. D. Hartman E. J., Keeler, "Layered neural networks with Gaussian hidden units as universal approximators " Neural computed., vol.2, $n^{\circ} .2$ (1990) 210-215

[4] - M. Salem, "Simple neuro-controller with a modified error function for a synchronous generator", International journal of electrical power and energy systems ,Vol. 25, $n^{\circ} .9$ (2003) 759-771

[5] - W. Liu, "Design of an adaptive neural network based power system stabilizer", Neural networks, Vol. 16, n5 (2003) 891-898

[6] - J. O. Jang And G. J. Jeon, "A parallel neuro-controller for DC motors containing nonlinear friction", Neurocomputing, Vol. 30, n¹ (2000) 233-248

[7] - L. Jin and M.M. Gupta, "Direct adaptive output tracking control using multilayer neural networks", IEEE Proc. Vol.140, nº (1993) 393-398 
[8] - A. Hajraoui, Commande numérique de la puissance électrique , Doctorat d'Etat, Fac. des sciences .Univ. Abdelmalek Essaadi, Tetouan, (1993) Maroc.

[9] - M. Gupta and H. Rao, " neuro-control systems : Theory and appliction", IEEE Press (1994) 11-24.

[10] - N. B. Karayiannis and venetsanopoulos, " Fast learning algorithms for neural networks", IEEE Trans. Cas.39, (1992) 453-474

[11] - B. Minaoui, " Contrôle de la puissance électrique par réseau de neurone", Document n³, (1996)

[12] - TMS320C3X DSP Starter Kit; Digital Signal Processing Solutions, Texas Instruments (1995)

[13] - B. Minaoui et A. Landi, "Etude et mise en œuvre d'un système de traitement numérique du signal en temps réel" Quatrième conférence internationale sur les mathématiques appliquées et les sciences de l'ingénieur. CIMASI (2002), actes du colloque, Casablanca, Maroc. 